\title{
Science of atmospheric phenomena with JEM-EUSO
}

\author{
The JEM-EUSO Collaboration
}

Received: 19 January 2014 / Accepted: 17 November 2014 / Published online: 2 July 2015

(C) The Author(s) 2015. This article is published with open access at Springerlink.com

\begin{abstract}
The main goal of the JEM-EUSO experiment is the study of Ultra High Energy Cosmic Rays (UHECR, $10^{19}-10^{21} e V$ ), but the method which will be used (detection of the secondary light emissions induced by cosmic rays in the atmosphere) allows to study other luminous phenomena. The UHECRs will be detected through the measurement of the emission in the range between 290 and $430 \mathrm{~m}$, where some part of Transient Luminous Events (TLEs) emission also appears. This work discusses the possibility of using the JEM-EUSO Telescope to get new scientific results on TLEs. The high time resolution of this instrument allows to observe the evolution of TLEs with great precision just at the moment of their origin. The paper consists of four parts: review of the present knowledge on the TLE, presentation of the results of the simulations of the TLE images in the JEM-EUSO telescope, results of the Russian experiment Tatiana-2 and discussion of the possible progress achievable in this field with JEM-EUSO as well as possible cooperation with other space projects devoted to the study of TLE - TARANIS and ASIM. In atmospheric
\end{abstract}

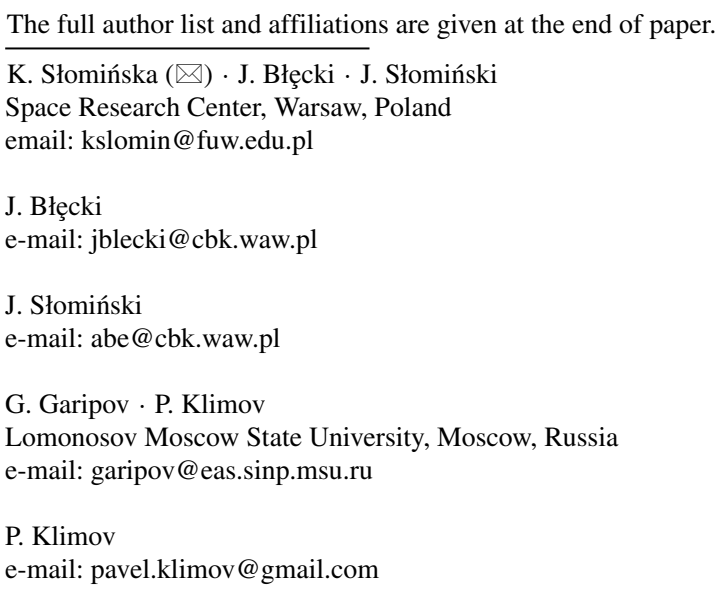


physics, the study of TLEs became one of the main physical subjects of interest after their discovery in 1989. In the years 1992 - 1994 detection was performed from satellite, aircraft and space shuttle and recently from the International Space Station. These events have short duration (milliseconds) and small scales ( $\mathrm{km}$ to tens of $\mathrm{km}$ ) and appear at altitudes $50-100 \mathrm{~km}$. Their nature is still not clear and each new experimental data can be useful for a better understanding of these mysterious phenomena.

Keywords Transient Luminous Events - Elves · Sprites - Jets - Atmospheric phenomena

\section{Introduction}

The Transient Luminous Events are bright, short-lived, atmospheric phenomena occurring above the clouds level, some of them reaching the ionosphere.

The first suggestion on the existence of that kind of phenomena was given by the Scottish physicists C.T.R. Wilson in 1925 [1], who observed that at a certain altitude above the clouds level the value of the electric field connected to the thunder cloud discharge equals the critical value of the electric field allowing for the conventional breakdown mechanism. This in turn means the production of light.

First confirmed observations of the light phenomena above clouds level date since 1989. The experimental discovery of one type of these events was made accidentally in 1989 by scientists from the University of Minnesota Robert Franz, Robert Nemzek and John Winckler. These first detections were related to a phenomenon, which now is called the red sprite. The observation was performed during night time, and only in 1998 Mark Stanley and his collaborators from New Mexico Tech observed a day time sprite [2]. Since then, thousands of observations of different types of TLEs have been carried out from aircrafts, balloons, space stations and satellites. Apart from red sprites, other types of TLE - blue jets, elves, halos and trolls - were observed. (Different types of TLE are schematically shown in the Fig. 1). According to all collected up to date observations, the TLEs are triggered by a significant electric discharge either inside a cloud or between the cloud and the Earth, which causes strong electromagnetic field above the cloud system. The electromagnetic field is a source of different light phenomena, some of them clasified as TLEs. It is estimated that the energy produced in the atmosphere by all types of TLEs is about $700 \mathrm{MJ}$ per minute [3]. In the case of sprites and elves the positive or negative cloud to Earth discharges are the necessary condition for this type of TLE to occur. Therefore, a strong correlation between the frequency of the thunderstorms and the sprites and elves is observed but the mechanism responsible for triggering the TLEs is still not clearly explained.

The wavelengths that these phenomena may produce depend on the composition of the atmosphere above the clouds - mainly Nitrogen - and on the allowed transitions observed in the molecules.

As JEM-EUSO detects wavelengths in the range 290 to $430 \mathrm{~nm}$, we will consider only the second positive line of Nitrogen with possible wavelenghts of $268-546 \mathrm{~nm}$. 


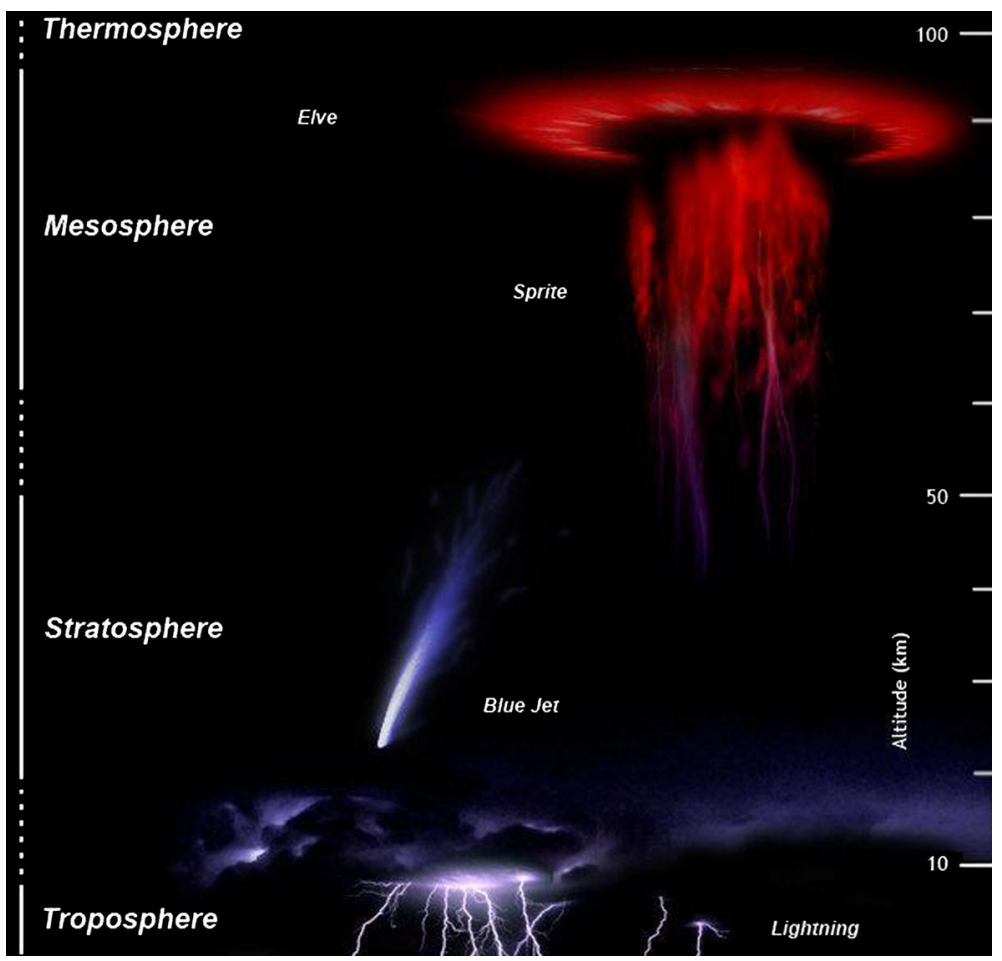

Fig. 1 A schematic overview of different types of the Transient Luminous Events. This picture comes from [4]

Other lines are either outside the visible spectrum of JEM-EUSO or their intensity is much lower than the second positive line of Nitrogen [5].

\section{JEM-EUSO in the TLE context}

\subsection{General description}

The JEM-EUSO mission is devoted to the observation of Extensive Air Showers (EAS) produced by UHECRs traversing the Earth's atmosphere from above. For each event, the detector will make accurate measurements of the energy, arrival direction and nature of the primary particle using a target volume far greater than what is achievable from ground [6]. The corresponding increase in statistics will help to clarify the origin and sources of UHECRs as well as to bring new light onto particle physics mechanisms responsible of production, propagation and acceleration.

\subsection{The detector}

JEM-EUSO will be attached to the Japanese Experiment Module (JEM) of the International Space Station (ISS) at an orbit of $400 \mathrm{~km}$ above the surface of the Earth. The 
JEM-EUSO detecting system consists of a telescope with three Fresnel lenses and a focal surface made of photomultipliers and associated electronics, which converts the photons collected by the lenses into photoelectrons. The focal surface consists of some $3 \cdot 10^{5}$ pixels, each with an independent read out. The diameter of the telescope aperture is about 2.5 meters. The instrument will work in two modes: the nadir mode and the tilted mode. It is planned to have the detector work in the nadir mode for 2 years and then in the tilted mode for further 3 years. The time unit used for sampling the data is 2.5 microseconds. The field of view (FoV) of the detector is $60^{\circ}$. The wavelengths measured by the detector may range from 290 to $430 \mathrm{~nm}$. In addition to this main telescope part, the detection system is completed by an Atmospheric Monitoring system consisting of an Infrared Camera and a LIDAR to provide information concerning the cloud top height. A calibration system, which measures the efficiencies of the optics, of the focal surface and the data acquisition system is provided as well.

\subsection{Triggering}

The photomultipliers of the JEM-EUSO detectors operate correctly with the total charge not exceeding $250 \mathrm{pC}$ per KI per time unit, which, in the case of JEMEUSO, is $2.5 \mu \mathrm{s}$, which is assumed to be the Gate Time Unit (GTU). A KI is a 4 by 2 pixels unit. Assuming the $10 \%$ efficiency of the optics and the electronics of the detector, this corresponds to $6.24 \cdot 10^{15}$ photoelectrons per KI per second, or $1.56 \cdot 10^{10}$ photoelectrons per KI per GTU. If this value is exceeded the voltage on the photomultipliers will be reduced so that the detection system remains safe.

\section{Estimation of TLE frequency for JEM-EUSO based on Tatiana-2 experimental data}

The global occurrence of TLE can be obtained in space-borne experiments. Only few detectors devoted to TLE measurements were launched to the orbit: ISUAL on board FORMOSAT-2 satellite [7], UV detector on board Universitetsky-Tatiana satellite [8], UV and IR detector on board Universitetsky-Tatiana-2 satellite [9] and JEMGLIMS on board ISS [10]. ISUAL and JEM-GLIMS have spatial resolution and provide spectral measurements in wide wavelength bands, but Tatiana-1 and Tatiana2 detectors provide measurements in $240-400 \mathrm{~nm}$, in the same wavelength range as EAS fluorescent radiation. It is important to mention that in both Tatiana experiments and in JEM-EUSO PMTs with UV filters are used to measure radiation. Oscillograms of fast luminous events are recorded. Tatiana-1 detector recorded only one flash (transient) event per orbit (because of poor transmission channel between satellite and ground receiving station) and obtained small statistics $(\approx 300$ TLE events during two years of operation). Tatiana-2 could measure the most powerful event per each minute during night part of orbit, which allowed to collect larger transient statistics (more than 2500 TLE events during three months). More details about detectors and their results are in $[11,12]$. Global distribution of all transient events, obtained by Tatiana-2 satellite is presented in the Fig. 2. The observations of TLE were conducted 


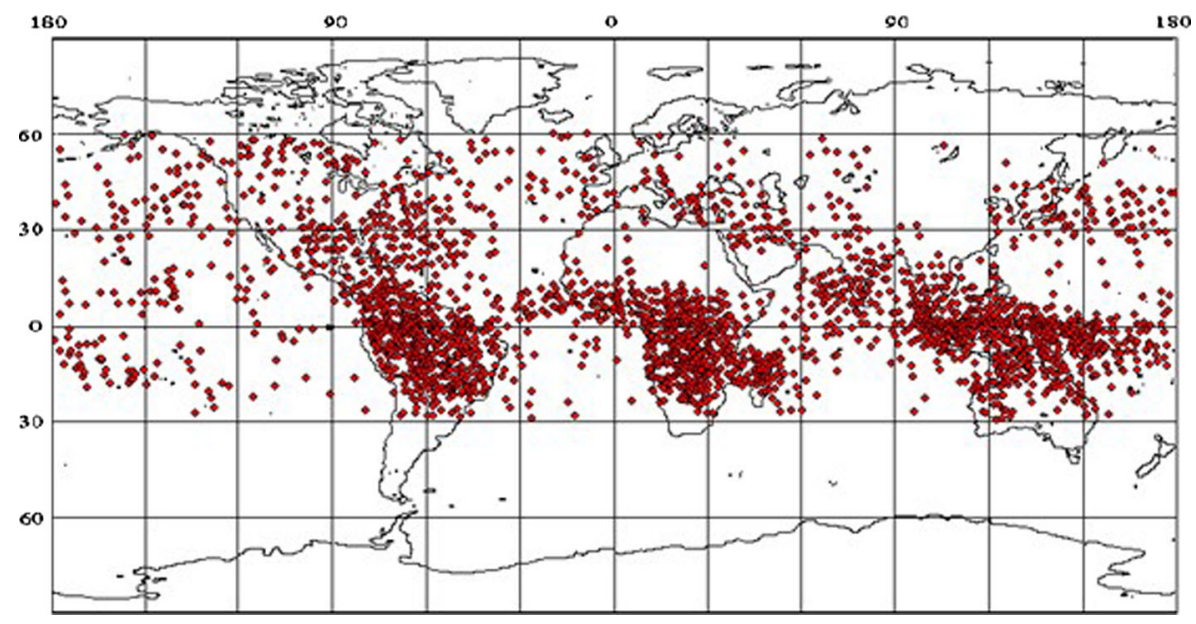

Fig. 2 Global distribution of all transient events

only on the night side of night part of the satellite orbit. The local night determines the distinct borders of observations: $60^{\circ}$ of latitude in the Northern hemisphere and $30^{\circ}$ in Southern hemisphere. Most of events are concentrated above continents in equatorial regions of America, Africa and Indo - China, this distribution seems similar to common lightning distribution. But the unexpected effect can be seen if we divide all events into groups with various energy ranges (number of photons). Events with smaller number of photons have more even geographical distribution and do not obviously correlate with huge thunderstorm regions (Fig. 3).

Tatiana's UV detectors have no spatial resolution and it is impossible to definitely distinguish types of TLE, to determine their actual size and spatial light emission distribution. However, the collected statistics allows us to estimate the low limit of transient event rate expected for space-borne detectors like JEM-EUSO and its influence on their duty cycle. In the period October 2009 - January 2010 Tatiana-2 detectors orbited the Earth 797 times with 320 hours of operation time in shadowed "night" part. During these three months 2628 events with $N_{A D C}>80$ were measured. The data of Tatiana-2 satellite of TLE events is recorded in a digital way and consists of $128 \mathrm{~ms}$ oscillograms of ADC code $\left(N_{A D C}\right)$ and PMT high voltage code $(M)$. ADC code means the signal amplitude and $M$ corresponds to a PMT gain. The average rate of TLE events from this data is $0.13 \mathrm{~min}^{-1}$ or $10^{-4} \mathrm{hr}^{-1} \mathrm{~km}^{-2}$. The instantaneous frequency of TLE in local thunderstorm area could be significantly higher $\left(\sim 10^{-3} \mathrm{hr}^{-1} \mathrm{~km}^{-2}\right)$. It is worth noticing that this number is order of magnitude higher than TLE frequency, measured by ISUAL $[12,13]$. It could be explained by the sensitivity of the detector whose instrumental threshold is $\sim 10^{20}$ in photon number. As it was mentioned above, these faint flashes are distributed more evenly in global map. ISUAL can not measure them.

To estimate the number of TLE events which could be measured by JEM-EUSO or influence its operation, we should take into account the ratio of detectors fields of view. Tatiana-2 observed an area of $7 \cdot 10^{4} \mathrm{~km}^{2}$, JEM-EUSO in nadir mode will 


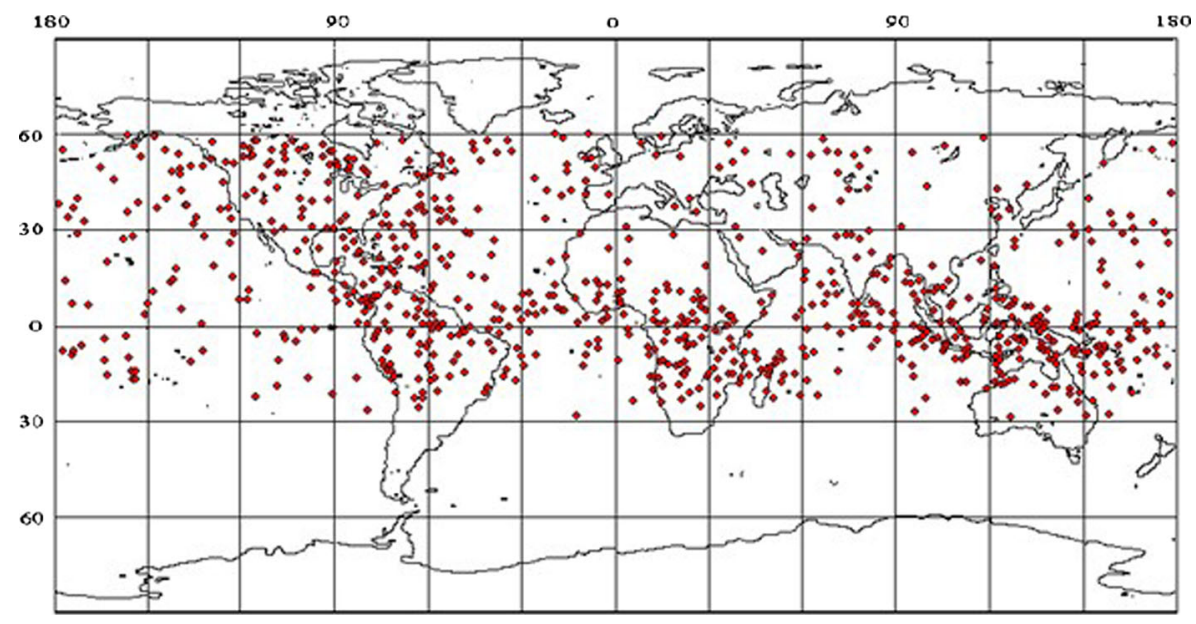

Fig. 3 Global map of transients with photon number less than $5 \cdot 10^{21}$ in the atmosphere

observe $2 \cdot 10^{5} \mathrm{~km}^{2}$, three times larger than Tatiana-2. It means that JEM-EUSO will measure one UV flash from atmospheric phenomena at least every third minute. In the worst case, when the size of TLE is comparable to JEM-EUSO FoV (for example in case of a large elve) or very intensive TLE which produces a huge amount of light scattered in detectors parts, it can cause a pause in EAS measurements for 70 seconds (it is the most conservative estimation of dead time within which the light source will leave JEM-EUSO FoV). Such dead time is $\sim 40 \%$ of the duty cycle. This is the upper bound. To obtain more feasible numbers we should take into account energy (number of photons) distribution of observed events.

The typical event measured by Tatiana-2 satellite has about $10^{4}$ photons per ms in $0.4 \mathrm{~cm}^{2}$ aperture of detector. It means that more than $10^{6}$ transient photons per GTU $(2.5 \mu s)$ will come in JEM-EUSO aperture $\left(4.5 \mathrm{~m}^{2}\right)$. The JEM-EUSO optics efficiency $(0.4)$ and the difference between orbit altitude of ISS $(\approx 400 \mathrm{~km})$ and Tatiana-2 satellite $(\approx 830 \mathrm{~km})$ were taken into account. In these calculations spatial light distribution in transients was not considered. Transient photons may be distributed among many pixels of photo receiver and signal in each pixel could be smaller. Actual size of TLE depends on its type. In case of elves this signal will be distributed among almost all pixels and will give few photoelectrons per GTU. Smaller events will significantly affect the operation of several specific Photo Detector Modules (PDM) and just slightly reduce the FoV.

The rest of the photo detector will be illuminated by diffuse light scattered in the detector (for our estimations we will consider diffuse light as $10 \%$ of the total number of photons per event). Diffuse light gives a signal increase of $\approx 0.5$ photoelectrons per GTU in one pixel. This is a minor addition to background signal which will not influence detector threshold and therefore a duty cycle. The fraction of flashes which produce more than 2 photoelectrons per GTU of scattered light in each pixel is $20 \%$ of all measured by Tatiana-2 satellite. If we consider 70 seconds 
pause only for these TLE the dead time becomes near $8 \%$. This number is consistent with other estimations of duty cycle which take into account clouds and thunderstorm regions.

\section{Estimation of the TLE light collected by the JEM-EUSO experiment}

\subsection{Elves}

The most common phenomena are ELVES - Emission of Light and VLF perturbations due to Electromagnetic Pulse Sources [14]. They occur at the lower ionosphere, at altitudes of about $90 \mathrm{~km}$. They are concentric rings of light expanding with speed close to the speed of light. The diameter of the elves disk may range from 200 to $500 \mathrm{~km}$. The whole process of expansion takes up to a millisecond.

The possible mechanism of production of an elve is as follows. Firstly, a strong lightning occurs - which translates into a rapid change in the electric field in the atmosphere. This produces an electromagnetic pulse propagating upwards through the atmosphere and eventually reaches the ionosphere where it causes production of light observed as an elve. To estimate the image viewed by the JEM-EUSO detector while observing an elve example, we have estimated the parameters of the average elve basing our assumptions on the results provided by the ISUAL experiment and presented in [3]. The occurrence rate of the elves is 35 per minute around the world. The spatially averaged brightness of an elve presented in [3] is: $0.17 \pm 0.08 M R=$ $1.36 \cdot 10^{14} \mathrm{ph} \mathrm{sr}^{-1} \mathrm{~m}^{-2} \mathrm{~s}^{-1}$. The unit $\mathrm{R}$ stands for "Rayleigh" (unit of photon flux) typically used for these atmospheric phenomena. We assume an elve with the diameter of $300 \mathrm{~km}$ and the timescale of $2 \mathrm{~ms}=800 \mathrm{GTU}$, which should allow for recording of the whole event and the observation directly in the JEM-EUSO detector in the nadir mode. This is the least favorable scenario in the sense that the geometry of the system allows for the maximum amount of light reaching the focal surface of the detector.

With all these assumptions, we get $9.25 \cdot 10^{11}$ photons, which is an estimate for the whole event, in all wavelength channels. As elves are ionospheric phenomena, they are mostly red. The contribution of "blue" wavelengths is marginal. If we take $13 \%$ of the obtained photons fitting our wavelength requirements (second positive line of Nitrogen), we will get the estimated number as: $1.2 \cdot 10^{11}$ photons. This number of photons will be recorded as a total number by all pixels of the focal surface throughout the duration of the event.

The number of pixels in the JEM-EUSO detector is 315 648, it equals to about 40000 KIs. The distribution of the photons among the separate KIs has not been considered here. Similarly, the time distribution of the incoming photons has not been taken into account in this work.

Both these factors will reduce the number of photons reaching a KI in one GTU by a few orders of magnitude. It is also important to note that the estimation relates to one such event and not to the total number of photons coming from other sources in the atmosphere. The calculations here presented are based on averaged results, while the most dangerous for the photomultipliers are the extreme cases of events 
producing light, for which the order of magnitude of the brightness may be a few hundred times greater.

The observed characteristics of different types of TLE can be found in the Table 1 .

\subsection{Sprites}

Sprites are associated with giant storm clouds with dimensions over $1000 \mathrm{~km}$ producing strong electric field in the mesosphere in a volume of over $10^{4} \mathrm{~km}^{3}$. Sprites are massive but weak luminous flashes, appearing at altitudes of $40-90 \mathrm{~km}$. The heads of sprites are predominantly red. The brightest region lies in the altitude range $65-75 \mathrm{~km}$, above which there is often a faint red glow structure that extends to about $90 \mathrm{~km}$. Below the bright red region, blue tendril-like filamentary structures often extend downward to $40 \mathrm{~km}$. Sprites rarely appear singly, usually occurring in groups - two, three or more. The duration of sprites is of the order of milliseconds. Currents of the lightning strokes associated with sprites reach the intensity of more than $100 \mathrm{kA}$ [17]. The pictures of sprites obtained from the ground and from aircraft show complex structures that assume a variety of forms. Early research reports for these events called them "upward lightning", "upward discharges", "cloud - to - stratosphere discharges" and "cloud - to - ionosphere discharges". Now they are called sprites. The tendril - streamer structure under the upper part of the phenomenon - the so called head of a sprite shows extremely interesting and as yet not fully explained phenomenon of merging streamers, which form bright

Table 1 Principal types of TLE in the upper atmosphere [16]

\begin{tabular}{|c|c|c|c|c|c|}
\hline $\begin{array}{l}\text { Type } \\
\text { of TLEs }\end{array}$ & $\begin{array}{l}\text { Altitude } \\
\text { Regime }\end{array}$ & $\begin{array}{l}\text { Transverse } \\
\text { Dimensions }\end{array}$ & $\begin{array}{l}\text { Spatial } \\
\text { Characteristics }\end{array}$ & $\begin{array}{l}\text { Apparent } \\
\text { Motion }\end{array}$ & Duration \\
\hline Sprites & $\sim 50-90 \mathrm{~km}$ & $\sim 1-20 \mathrm{~km}$ & $\begin{array}{l}\text { Top }(>80 \mathrm{~km}) \\
\text { diffuse } \\
\text { Bottom }(<70 \\
\mathrm{km}) \\
\text { structured }\end{array}$ & $\begin{array}{l}\text { Top - } \\
\text { upward } \\
\text { Bottom - } \\
\text { downward }\end{array}$ & few ms \\
\hline Elves & $\sim 90 \mathrm{~km}$ & $>100 \mathrm{~km}$ & Diffuse & $\begin{array}{l}\text { Lateral } \\
\text { expansion }\end{array}$ & $\sim \mathrm{ms}$ \\
\hline $\begin{array}{l}\text { Blue jets } \\
\text { Giant } \\
\text { Blue jets }\end{array}$ & $\begin{array}{l}\sim 18-45 \mathrm{~km} \\
\sim 18-75 \mathrm{~km}\end{array}$ & $\begin{array}{l}\text { few km } \\
\text { few km }\end{array}$ & $\begin{array}{l}\text { Structured } \\
\text { Structured }\end{array}$ & $\begin{array}{l}\text { Upward } \\
\text { Upward }\end{array}$ & $\begin{array}{l}\text { hundreds ms } \\
\text { hundreds ms }\end{array}$ \\
\hline Hallos & $\sim 75 \mathrm{~km}$ & $50 \mathrm{~km}$ & Diffuse & Downward & $\sim \mathrm{ms}$ \\
\hline Trolls & $\sim 60-70 \mathrm{~km}$ & $\mathrm{kms}$ & Structured & $\begin{array}{l}\text { Upward } \\
\text { within } \\
\text { decaying } \\
\text { sprite } \\
\text { tendrils }\end{array}$ & hundreds ms \\
\hline
\end{tabular}


spherical structures - the beads. Measurements of microsecond time resolution may help to explain the mechanism behind the phenomenon. The brightness measured by ISUAL is $1.5 \pm 1.1 \mathrm{MR}$, which translates into some $3.48 \cdot 10^{11} \mathrm{ph}$ in total and $1.46 \cdot 10^{11}$ in the wavelength region detected by the JEM-EUSO. This number is the spatially integrated amount of photons recorded throughout the whole event duration.

\subsection{Other types of TLE}

Blue Jets Blue jets are optical ejections from the top of the electrically most active regions of thunderstorms. Following their emergence from the top of the thundercloud, they typically propagate upward in narrow structure in form of cones of about 15 degrees full width at vertical speeds of roughly $100 \mathrm{~km} / \mathrm{s}$, fanning out and disappearing at heights of about $40-50 \mathrm{~km}$. At the base their diameter is about 400 $\mathrm{m}$. Blue jets are rather rare events. Their appearance rate is much lower than that of sprites. Blue jets are not aligned with the local magnetic field. They are triggered by the electric field inside the cloud. Their appearance is not connected to a cloud-to-ground discharge. The brightness of blue jets is estimated as 0.5 MR [15].

Gigantic Jets They are much larger than the Blue Jets. Gigantic Jets originate form the cloud top and develop into a tree-like structure reaching the lower ionosphere. Their propagate upward at the speed of about $100 \mathrm{~km} / \mathrm{s}$. The ISUAL measured only 8 such events. The brightest one was $0.8 \mathrm{MR}$.

\section{Conclusions and the future}

The frequency of occurrence of the TLE events is large. Their characteristics have been described by different groups working with detectors into orbit and with on ground detecting systems. JEM-EUSO is unique in that its time and spatial resolution is very high and will allow time evolution measurement. Two more experiments dedicated to measuring TLEs will soon be put into orbit: ASIM and Taranis. The set of data produced by all these experiments may create an exceptional chance to further investigate the spatial and time evolution of the TLEs.

The amount of light produced by the two most common types of TLEs does not pose danger to the photomultipliers of the detector. However, these phenomena constitute a significant background to the detection of UHECRs. Many aspects of the detection of the signal from the TLEs remain to be considered in next developments of this work. One interesting path would also be to test the possibility of finding correlations between the occurrence of very energetic particles in the Earth's atmosphere and the occurrence and magnitude of sprites. These goals are in the reach of the JEM - EUSO mission in space and, thoghether with its unique features above mentioned, they may lead to significant steps forward in the field of Transient Luminous Events research.

Acknowledgments This work is supported partially by the grant MNiSW N307065834 and grant NCN 2014/13/B/ST10/01285 and grant of Russian Foundation for Basic Research No. 12-05-31025-mol-a. Authors wish to thank all involved in the preparation and the reviewing process of this paper. 
Open Access This article is distributed under the terms of the Creative Commons Attribution License which permits any use, distribution, and reproduction in any medium, provided the original author(s) and the source are credited.

\section{References}

1. Wilson, C.T.R.: The electric field of a thundercloud and some of its effects. Proc. Phys. Soc. London 37, 32D (1925)

2. Schneider, D.: Sci. Am. 88(4), 1 (2000)

3. Kuo, C.L., et al.: Radiative emission and energy deposition in transient luminous events. J. Phys. D: Appl. Phys. 41, 234014 (14pp) (2008)

4. http://upload.wikimedia.org/wikipedia/commons/c/c4/Upperatmoslight1.jpg

5. Kuo, C.L., et al.: Modeling elves observed by FORMOSAT-2 satellite. J. Geophys. Res. 112, A11312 (2007). doi:10.1029/2007JA012407

6. Adams Jr. J.H., et al. (JEM-EUSO Collaboration): An evaluation of the exposure in nadir observation of the JEM-EUSO mission. Astro. Phys. 44, 76-90 (2013)

7. Chern, J.L., Hsu, R.R., Su, H.T., Mende, S.B., Fukunishi, H., Takahashi, Y., Lee, L.C.: Global survey of upper atmospheric transient luminous events on the ROCSAT-2 satellite. J. Atmos. Sol. Terr. Phys. 65(5), 647-659 (2003)

8. Garipov, G.K., Panasyuk, M.I., Rubinshtein, I.A., et al.: UV detector onboard research and education microsatellite Universitetsky-Tatiana of Moscow University. Prib. Tekh. Eksp. 1, 135-141 (2006)

9. Sadovnichy, V.A., Panasyuk, M.I., Yashin, I.V., et al.: Investigation of space environment aboard Universitetsky-Tatiana and Universitetsky-Tatiana-2 microsatellites. Solar System Res. 45(1), 3-29 (2011)

10. Sato, M., Takahashi, Y., Kikuchi, M., Suzuki, M., Yamazaki, A., Ushio, T.: Lightning and sprite imager (LSI) onboard JEM-GLIMS. IEEJ Trans. FM 131(12), 994-999 (2011)

11. Garipov, G.K., Khrenov, B.A., Panasyuk, M.I., et al.: UV radiation from the atmosphere: results of the MSU Tatiana satellite measurements. Astropart. Phys. 24, 400-408 (2005)

12. Vedenkin, N.N., Garipov, G.K., Klimov, P.A., Klimenko, V.V., Mareev, E.A., Martinez, O., Morozenko, V.S., Park, I.H., Panasyuk, M.I., Salazar, U., Tulupov, V.I., Khrenov, B.A., Yashin, I.V.: Atmospheric ultraviolet and red infrared flashes from universitetsky Tatiana-2 satellite data. JETP 113(5), ISSN 781-790 (2011)

13. Chen, A.B., Kuo, C.L., et al.: Global distribution and occurrence rate of TLE. JGR 118, A0806 (2008). doi:101029/029/2008JAO013101

14. Fukunishi, H., et al.: Elves: Lightning-induced transient luminous events in the lower ionosphere. Geophys. Res. Let. 23, 2157-2160 (1996)

15. Wescott, E.M., Sentman, D., et al: Preliminary results from the Sprites 94 aircraft campaign: 2. Blue jets. Geophys. Res. Lett. 22(10), 1205-1208 (1995)

16. Sentman, D.D.: Overview of transient luminous events, presentation at space science laboratory seminar. University of Berkeley, California (2005). -updated for this paper

17. Füllerkrug, M., Mareev, E.A., Rycroft, M.J.: Sprites, elves and intense lightning discharges - book, NATO science series (2006)

\section{The JEM-EUSO Collaboration}

J.H. Adams Jr. ${ }^{m d}$, S. Ahmad ${ }^{b b}$, J.-N. Albert ${ }^{b a}$, D. Allard ${ }^{b c}$, L. Anchordoqui ${ }^{m f}$, V. Andreev Ane $^{m}$, A. Anzalone ${ }^{d h, d n}$, Y. Arai ${ }^{e v}$, K. Asano ${ }^{e t}$, M. Ave Pernas ${ }^{k c}$, P. Baragatti ${ }^{d o}$,P. Barrillon ${ }^{b a}$, T. Batsch ${ }^{h c}$, J. Bayer ${ }^{c d}$, R. Bechinid ${ }^{d l}$, T. Belenguer ${ }^{k b}$, R. Bellotti ${ }^{d a}, d b$, K. Belov ${ }^{m e}$, A.A. Berlind ${ }^{m h}$, M. Bertaina ${ }^{d k, d l}$, P.L. Biermann ${ }^{c b}$, S. Biktemerova ${ }^{i a}$, C. Blaksley ${ }^{b c}$, N. Blanc ${ }^{l a}$, J. Błęcki ${ }^{h d}$, S. Blin-Bondil ${ }^{b b}$, J. Blümer ${ }^{c b}$, P. Bobik ${ }^{j a}$, M. Bogomilov ${ }^{a a}$, M. Bonamente ${ }^{m d}$, M.S. Briggs ${ }^{m d}$, S. Briz ${ }^{k d}$, A. Bruno ${ }^{d a}$, F. Cafagna ${ }^{d a}$, D. Campana ${ }^{d f}$, J-N. Capdevielle ${ }^{b c}$, R. Caruso ${ }^{d c, d n}$, M. Casolino ${ }^{e w, d i}$, C. Cassardo ${ }^{d k, d l}$, 
G. Castellini $^{d d}$, C. Catalano ${ }^{b d}$, O. Catalano ${ }^{d h, d n}$, A. Cellino ${ }^{d k, d m}$, M. Chikawa ${ }^{e d}$, M.J. Christ ${ }^{m g}$,

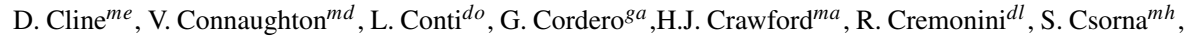
S. Dagoret-Campagne ${ }^{b a}$, A.J. de Castro ${ }^{k d}$, C. De Donato ${ }^{d i}$, C. de la Taille ${ }^{b b}$, C. De Santis ${ }^{d i, d j}$, L. del Peral ${ }^{k c}$, A. Dell'Oro ${ }^{d k, d m}$ N. De Simone ${ }^{d i}$, M. Di Martino ${ }^{d k, d m}$, G. Distratis ${ }^{c d}$, F. Dulucq ${ }^{b b}$, M. Dupieux ${ }^{b d}$, A. Ebersoldt ${ }^{c b}$, T. Ebisuzaki ${ }^{e w}$, R. Engel ${ }^{c b}$, S. Falk ${ }^{c b}$, K. Fang ${ }^{m b}$, F. Fenu ${ }^{c d}$, I. FernándezGómez $^{k d}$, S. Ferrarese ${ }^{d k, d l}$, D. Finco ${ }^{d o}$, M. Flamini ${ }^{d o}$, C. Fornaro ${ }^{d o}$, A. Franceschid ${ }^{d e}$, J. Fujimoto ${ }^{e v}$, M. Fukushima ${ }^{e g}$, P. Galeotti ${ }^{d k, d l}$, G. Garipov ${ }^{i c}$, J. Geary ${ }^{m d}$, G. Gelmini $^{m e}$, G. Giraudo $^{d k}$, M. Gonchar $^{i a}$, C. González Alvarado ${ }^{k b}$, P. Gorodetzky ${ }^{b c}$, F. Guarino ${ }^{d f, d g}$, A. Guzmán ${ }^{c d}$, Y. Hachisu ${ }^{e w}$, B. Harlov ${ }^{i b}$, A. Haungs ${ }^{c b}$, J. Hernández Carretero ${ }^{k c}$, K. Higashide ${ }^{e r, e w}$, D. Ikeda ${ }^{e g}, H$. Ikeda ${ }^{e p}$, N. Inoue ${ }^{e r}$, S. Inoue ${ }^{e g}$, A. Insolia ${ }^{d c, d n}$, F. Isgrò̀ ${ }^{d f, d p}$, Y. Itow ${ }^{e n}$, E. Joven ${ }^{k e}$, E.G. Judd ${ }^{m a}$, A. Jung ${ }^{f b}$, F. Kajino ${ }^{e i}$, T. Kajino ${ }^{e l}$, I. Kaneko ${ }^{e w}$, Y. Karadzhov ${ }^{a a}$, J. Karczmarczyk ${ }^{h c}$, M. Karus ${ }^{c b}$, K. Katahira ${ }^{e w}$, K. Kawaiew, Y. Kawasaki ${ }^{e w}$, B. Keilhauer ${ }^{c b}$, B.A. Khrenov ${ }^{i c}$, Jeong-Sook $\operatorname{Kim}^{f a}$, Soon-Wook Kim ${ }^{f a}$, SugWhan Kim ${ }^{f d}$, M. Kleifges ${ }^{c b}$, P.A. Klimov ${ }^{i c}$, D. Kolev ${ }^{a a}$, I. Kreykenbohm ${ }^{c a}$, K. Kudela ${ }^{j a}$, Y. Kurihara ${ }^{e v}$, A. Kusenko ${ }^{m e}$, E. Kuznetsov ${ }^{m d}$, M. Lacombe ${ }^{b d}$, C. Lachaud ${ }^{b c}$, J. Lee ${ }^{f c}$, J. Licandro ${ }^{k e}, \mathrm{H}_{\text {L Lim }}{ }^{f c}$, F. López ${ }^{k d}$, M.C. Maccarone ${ }^{d h, d n}$, K. Mannheim ${ }^{c e}$, D. Maravilla ${ }^{g a}$, L. Marcelli ${ }^{d j}$, A. Marini ${ }^{d e}$, O. Martinez ${ }^{g c}$, G. Masciantonio ${ }^{d i, d j}$, K. Mase ${ }^{e a}$, R. Matev ${ }^{a a}$, G. Medina-Tanco ${ }^{g a}$, T. Mernik ${ }^{c d}$, H. Miyamoto ${ }^{b a}$, Y. Miyazaki ${ }^{e c}$, Y. Mizumoto ${ }^{e l}$, G. Modestino ${ }^{d e}$, A. Monaco ${ }^{d a}, d b$, D. MonnierRagaigne $^{b a}$, J.A. Morales de los Ríos ${ }^{k a, k c}$, C. Moretto ${ }^{b a}$, V.S. Morozenko ${ }^{i c}$, B. Mot ${ }^{b d}$, T. Murakami ${ }^{e f}$, M. Nagano ${ }^{e c}$, M. Nagata ${ }^{e h}$, S. Nagataki ${ }^{e k}$, T. Nakamura ${ }^{e j}$, T. Napolitano ${ }^{d e}$, D. Naumov ${ }^{i a}$, R. Nava ${ }^{g a}$, A. Neronov ${ }^{l b}$, K. Nomoto ${ }^{e u}$, T. Nonaka ${ }^{e g}$, T. Ogawa ${ }^{e w}$, S. Ogio ${ }^{e o}$, H. Ohmorie ${ }^{e w}$, A.V. Olinto ${ }^{m b}$, P. Orleański ${ }^{h d}$, G. Osteria ${ }^{d f}$, M.I. Panasyuk ${ }^{i c}$, E. Parizot ${ }^{b c}$, I.H. Park ${ }^{f c}$, H.W. Park ${ }^{f c}$, B. Pastircak ${ }^{j a}{ }^{\text {, }}$ T. Patzak ${ }^{b c}$, T. Paul ${ }^{m f}$, C. Pennypacker ${ }^{m a}$, S. Perez Cano ${ }^{k c}$, T. Peter ${ }^{l c}$, P. Picozza ${ }^{d i, d j, e w}$, T. Pierog ${ }^{c b}$, L.W. Piotrowski ${ }^{e w}$, S. Piraino ${ }^{c d, d h}$, Z. Plebaniak ${ }^{h c}$, A. Pollini ${ }^{l a}$, P. Prat ${ }^{b c}$, G. Prévôt ${ }^{b c}$, H. Prieto ${ }^{k c}$, M. Putis ${ }^{j a}$, P. Reardon ${ }^{m d}$, M. Reyes ${ }^{k e}$, M. Ricci ${ }^{d e}$, I. Rodríguez ${ }^{k d}$, M.D. Rodríguez Frías ${ }^{k c}$, F. Ronga ${ }^{d e}$, M. Roth ${ }^{c b}$, H. Rothkaeh ${ }^{h d}$, G. Roudil ${ }^{b d}$, I. Rusinov ${ }^{a a}$, M. Rybczyński ${ }^{h a}$, M.D. Sabau ${ }^{k b}$, G. Sáez Cano ${ }^{k c}$, H. Sagawa ${ }^{e g}$, A. Saito ${ }^{e j}$, N. Sakaki ${ }^{c b}$, M. Sakata ${ }^{e i}$, H. Salazar ${ }^{g c}$, S. Sánchez ${ }^{k d}$, A. Santangelo ${ }^{c d}$, L. Santiago Crúz ${ }^{g a}$, M. Sanz Palomino ${ }^{k b}$, O. Saprykin ${ }^{i b}$, F. Sarazin ${ }^{m c}$, H. Sato ${ }^{e i}$, M. Sato ${ }^{e s}$, T. Schanz ${ }^{c d}$, H. Schieler ${ }^{c b}$, V. Scottid ${ }^{d f, d g}$, A. Segreto ${ }^{d h, d n}$, S. Selmane ${ }^{b c}$, D. Semikoz ${ }^{b c}$, M. Serra ${ }^{k e}$, S. Sharakin ${ }^{i c}$, T. Shibata ${ }^{e q}$, H.M. Shimizu ${ }^{e m}$, K. Shinozaki ${ }^{e w, c d}$, T. Shirahama ${ }^{e r}$, G. Siemieniec-Oziębło ${ }^{h b}$, H.H. Silva López ${ }^{g a}$, J. Sledd $^{m g}$, K. Słomińska ${ }^{h d}{ }^{\text {A. A. Sobey }}{ }^{m g}$, T. Sugiyama $^{e m}$, D. Supanitsky ${ }^{g a}$, M. Suzuki ${ }^{e p}$, B. Szabelska ${ }^{h c}$, J. Szabelski ${ }^{h c}$, F. Tajima ${ }^{e e}$, N. Tajima ${ }^{e w}$, T. Tajima ${ }^{c c}$, Y. Takahashi ${ }^{e s}$, H. Takami ${ }^{e v}$, M. Takeda ${ }^{e g}$, Y. Takizawa ${ }^{e w}$, C. Tenzer ${ }^{c d}$, O. Tibolla ${ }^{c e}$, L. Tkachev $^{i a}$, H. Tokuno ${ }^{e t}$, T. Tomida ${ }^{e w}$, N. Tone ${ }^{e w}$, S. Toscano ${ }^{l b}$, F. Trillaud ${ }^{g a}$, R. Tsenov ${ }^{a a}$, Y. Tsunesada ${ }^{e t}$, K. Tsuno ${ }^{e w}$, T. Tymieniecka ${ }^{h c}$, Y. Uchihori ${ }^{e b}$, M. Unger ${ }^{c b}$, O. Vaduvescu ${ }^{k e}$, J.F. Valdés-Galiciag ${ }^{g a}$, P. Vallania ${ }^{d k, d m}$, L. Valore ${ }^{d f, d g}$, G. Vankova ${ }^{a a}$, C. Vigorito ${ }^{d k, d l}$, L. Villaseñor ${ }^{g b}$, P. von Ballmoos ${ }^{b d}$, S. Wada ${ }^{e w}$, J. Watanabe ${ }^{e l}$, S. Watanabe ${ }^{e s}$, J. Watts Jr. ${ }^{m d}$, M. Weber ${ }^{c b}$, T.J. Weiler ${ }^{m h}$, T. Wibig ${ }^{h c}$, L. Wiencke ${ }^{m c}$, M. Wille ${ }^{c a}$, J. Wilms ${ }^{c a}$, Z. Włodarczyk ${ }^{h a}$, T. Yamamoto ${ }^{e i}$, Y. Yamamoto ${ }^{e i}$, J. Yang ${ }^{f b}$, H. Yano ${ }^{e p}$, I.V. Yashin ${ }^{i c}$, D. Yonetoku ${ }^{e f}$, K. Yoshida ${ }^{e i}$, S. Yoshida ${ }^{e a}$, R. Young ${ }^{m g}$, M.Yu. Zotov ${ }^{i c}$, A. Zuccaro Marchi ${ }^{e w}$

${ }^{a}$ St. Kliment Ohridski University of Sofia, Bulgaria

${ }^{b a}$ LAL, Univ Paris-Sud, CNRS/IN2P3, Orsay, France

${ }^{b b}$ Omega, Ecole Polytechnique, CNRS/IN2P3, Palaiseau, France

${ }^{b c}$ APC, Univ Paris Diderot, CNRS/IN2P3, CEA/Irfu, Obs. de Paris, Sorbonne Paris Cité, France

${ }^{b d}$ IRAP, Université de Toulouse, CNRS, Toulouse, France

${ }^{c a}$ ECAP, University of Erlangen-Nuremberg, Germany

${ }^{c b}$ Karlsruhe Institute of Technology (KIT), Germany

${ }^{c c}$ Ludwig Maximilian University, Munich, Germany

${ }^{c d}$ Inst. for Astronomy and Astrophysics, Kepler Center, University of Tübingen, Germany

${ }^{c e}$ Institut für Theoretische Physik und Astrophysik, University of Würzburg, Germany

${ }^{d a}$ Istituto Nazionale di Fisica Nucleare - Sezione di Bari, Italy

${ }^{d b}$ Universita' degli Studi di Bari Aldo Moro and INFN - Sezione di Bari, Italy

${ }^{d c}$ Dipartimento di Fisica e Astronomia - Universita' di Catania, Italy

${ }^{d d}$ Consiglio Nazionale delle Ricerche (CNR) - Ist. di Fisica Applicata Nello Carrara, Firenze, Italy

de Istituto Nazionale di Fisica Nucleare - Laboratori Nazionali di Frascati, Italy

$d f$ Istituto Nazionale di Fisica Nucleare - Sezione di Napoli, Italy

${ }^{d g}$ Universita' di Napoli Federico II - Dipartimento di Scienze Fisiche, Italy 
${ }^{d h}$ INAF - Istituto di Astrofisica Spaziale e Fisica Cosmica di Palermo, Italy

${ }^{d i}$ Istituto Nazionale di Fisica Nucleare - Sezione di Roma Tor Vergata, Italy

${ }^{d j}$ Universita' di Roma Tor Vergata - Dipartimento di Fisica, Roma, Italy

${ }^{d k}$ Istituto Nazionale di Fisica Nucleare - Sezione di Torino, Italy

${ }^{d l}$ Dipartimento di Fisica, Universita' di Torino, Italy

${ }^{d m}$ Osservatorio Astrofisico di Torino, Istituto Nazionale di Astrofisica, Italy

${ }^{d n}$ Istituto Nazionale di Fisica Nucleare - Sezione di Catania, Italy

${ }^{d o}$ UTIU, Dipartimento di Ingegneria, Rome, Italy

${ }^{d p}$ DIETI, Universita' degli Studi di Napoli Federico II, Napoli, Italy

ea Chiba University, Chiba, Japan

${ }^{e b}$ National Institute of Radiological Sciences, Chiba, Japan

$e^{e c}$ Fukui University of Technology, Fukui, Japan

ed Kinki University, Higashi-Osaka, Japan

ee Hiroshima University, Hiroshima, Japan

ef Kanazawa University, Kanazawa, Japan

eg Institute for Cosmic Ray Research, University of Tokyo, Kashiwa, Japan

eh Kobe University, Kobe, Japan

ei Konan University, Kobe, Japan

ej Kyoto University, Kyoto, Japan

ek Yukawa Institute, Kyoto University, Kyoto, Japan

${ }^{e l}$ National Astronomical Observatory, Mitaka, Japan

em Nagoya University, Nagoya, Japan

en Solar-Terrestrial Environment Laboratory, Nagoya University, Nagoya, Japan

${ }^{e o}$ Graduate School of Science, Osaka City University, Japan

$e p$ Institute of Space and Astronautical Science/JAXA, Sagamihara, Japan

eq Aoyama Gakuin University, Sagamihara, Japan

er Saitama University, Saitama, Japan

es Hokkaido University, Sapporo, Japan

${ }^{e t}$ Interactive Research Center of Science, Tokyo Institute of Technology, Tokyo, Japan

eu University of Tokyo, Tokyo, Japan

ev High Energy Accelerator Research Organization (KEK), Tsukuba, Japan

ew RIKEN, Wako, Japan

$f a$ Korea Astronomy and Space Science Institute (KASI), Daejeon, Republic of Korea

${ }^{f b}$ Ewha Womans University, Seoul, Republic of Korea

${ }^{f c}$ Sungkyunkwan University, Seoul, Republic of Korea

${ }^{f d}$ Center for Galaxy Evolution Research, Yonsei University, Seoul, Republic of Korea

${ }^{g a}$ Universidad Nacional Autónoma de México (UNAM), Mexico

${ }^{g b}$ Universidad Michoacana de San Nicolas de Hidalgo (UMSNH), Morelia, Mexico

${ }^{g c}$ Benemérita Universidad Autónoma de Puebla (BUAP), Mexico

ha Jan Kochanowski University, Institute of Physics, Kielce, Poland

$h b$ Jagiellonian University, Astronomical Observatory, Krakow, Poland

${ }^{h c}$ National Centre for Nuclear Research, Lodz, Poland

hd Space Research Centre of the Polish Academy of Sciences (CBK), Warsaw, Poland

ia Joint Institute for Nuclear Research, Dubna, Russia

${ }^{i b}$ Central Research Institute of Machine Building, TsNIIMash, Korolev, Russia

${ }^{i c}$ Skobeltsyn Institute of Nuclear Physics, Lomonosov Moscow State University, Russia

${ }^{j a}$ Institute of Experimental Physics, Kosice, Slovakia

${ }^{k a}$ Consejo Superior de Investigaciones Científicas (CSIC), Madrid, Spain

${ }^{k b}$ Instituto Nacional de Técnica Aeroespacial (INTA), Madrid, Spain

${ }^{k c}$ Universidad de Alcalá (UAH), Madrid, Spain

$k d$ Universidad Carlos III de Madrid, Spain

ke Instituto de Astrofísica de Canarias (IAC), Tenerife, Spain

la Swiss Center for Electronics and Microtechnology (CSEM), Neuchâtel, Switzerland

${ }^{l b}$ ISDC Data Centre for Astrophysics, Versoix, Switzerland

${ }^{l c}$ Institute for Atmospheric and Climate Science, ETH Zürich, Switzerland

$m a$ Space Science Laboratory, University of California, Berkeley, USA

$m b$ University of Chicago, USA 
${ }^{m c}$ Colorado School of Mines, Golden, USA

${ }^{m d}$ University of Alabama in Huntsville, Huntsville, USA

me University of California (UCLA), Los Angeles, USA

${ }^{m f}$ University of Wisconsin-Milwaukee, Milwaukee, USA

${ }^{m g}$ NASA - Marshall Space Flight Center, USA

${ }^{m h}$ Vanderbilt University, Nashville, USA 